\title{
Correction objectives have higher impact than screw pattern and density on the optimal 3D correction of thoracic AIS: a biomechanical study
}

\author{
Luigi La Barbera ${ }^{1,2,4}$ (D) A. Noelle Larson ${ }^{3} \cdot$ Carl-Eric Aubin $^{1,2}$
}

Received: 11 July 2020 / Accepted: 14 December 2020 / Published online: 26 January 2021

(c) Crown 2021

\begin{abstract}
Study design Assessment of screw pattern, implant density (ID), and optimization of 3D correction through computer-based biomechanical models.

Objective To investigate how screw pattern and ID affect intraoperative 3D correction of thoracic curves in adolescent idiopathic scoliosis, and how different correction objectives impact the optimal screw pattern.

Summary of background data Screw pattern, ID, correction objectives and surgical strategies for posterior fusion of AIS are highly variable among experienced surgeons. The "optimal" instrumentation remains not well defined.

Methods 10 patient-specific multibody models of representative adolescent idiopathic scoliosis Lenke 1A cases were built and used to compare alternative virtual correction surgeries. Five screw patterns and IDs (average: 1.6 screws/instrumented level, range: 1.2-2) were simulated, considering concave rod rotation, en bloc derotation, and compression/distraction as primary correction maneuvers. 3D correction descriptors were quantified in the coronal, sagittal and transverse planes. An objective function weighting the contribution of intraoperative 3D correction and mobility allowed rating of the outcomes of the virtual surgeries. Based on surgeon-dependent correction objectives, the optimal result among the simulated constructs was identified.

Results Low-density (ID $\leq 1.4$ ) constructs provided equivalent 3D correction compared to higher (ID $\geq 1.8$ ) densities (average differences ranging between $2^{\circ}$ and $3^{\circ}$ ). The optimal screw pattern varied from case to case, falling within the low-density screw category in $14 \%$ of considered scenarios, $73 \%$ in the mid-density $(1.4<$ ID $<1.8)$ and $13 \%$ in the high-density. The optimal screw pattern was unique in five cases; multiple optima were found in other cases depending on the considered correction objectives.

Conclusions Low-density screw patterns provided equivalent intraoperative 3D correction to higher-density patterns. Simulated surgeon's choice of correction objectives had the greatest impact on the selection of the optimal construct for 3D correction, while screw density and ID had a limited impact.
\end{abstract}

Level of evidence N/A.

Keywords Adolescent idiopathic scoliosis (AIS) $\cdot$ Correction objectives $\cdot$ Screw pattern $\cdot$ Implant density $\cdot$ Multibody model - Optimization · Patient-specific biomechanical modeling

Luigi La Barbera

luigi.labarbera@polimi.it

1 Department of Mechanical Engineering, Polytechnique Montreal, Downtown Station, P.O. Box 6079, Montreal, QC H3C 3A7, Canada

2 Research Center, Sainte-Justine University Hospital Center, 3175, Cote Sainte-Catherine Road, Montreal, QC H3T 1C5, Canada
3 Department of Orthopedic Surgery, Mayo Clinic, Rochester, MN, USA

4 Laboratory of Biological Structure Mechanics, Department of Chemistry, Materials and Chemical Engineering, Giulio Natta, Politecnico di Milano, Piazza Leonardo da Vinci 32, 20133 Milano, Italy 


\section{Introduction}

The treatment of adolescent idiopathic scoliosis (AIS) through posterior instrumented fusion has significantly evolved in the last decades. Since the introduction of pedicle screws, correction techniques have continuously evolved, allowing powerful control on 3D spine shape through a variable number of anchoring points [1]. Screw pattern and implant density (ID, defined as the number of implants per vertebra over the instrumented spine segment) may be expected to affect the selection of surgical maneuvers to achieve adequate 3D correction. Although these aspects are certainly related, the high inter-surgeons variability (ID range 1-2) indicates that there is no consensus [2-13]. Considering that high implant density has been associated with increased surgery time, blood loss, complications and costs $[14,15]$, using fewer implants may be beneficial. While previous retrospective studies are underpowered to confirm the clinical advantages of decreased implant density [15-17], a recent randomized controlled trial (RCT) by the Minimize Implants Maximize Outcomes (MIMO) Study Group demonstrated that equivalent correction in the coronal plane could be achieved using lower IDs [18].

3D correction objectives are defined as the choice to target one or more specific descriptors of spinal deformity on different anatomical planes (i.e., Cobb angle, transverse plane vertebral rotation, thoracic kyphosis) using specific maneuvers. Surgeon preference regarding correction objectives are believed to be responsible for the high variability among surgeons and may affect the final outcome [2, 19, 20]. The lack of standardization regarding surgical techniques affected by variable surgeon experience, knowledge and perspective of the complex biomechanics of 3D spine deformity correction is an additional issue [21].

Patient-specific computer-based surgical simulations are a valuable tool to virtually test the impact of a variety of screw patterns, densities and techniques on 3D correction in a finely controlled environment excluding confounding factors [2, 21-24]. Using such tools, Wang et al. did not find significant differences of correction with different implant densities (ID) following simulated concave rod rotation and compression/distraction correction maneuvers [24]. Martino et al. reported improved transverse plane correction using a maximal density $(I D=2)$ construct compared to a very low-density one (ID $=1)$, following concave rod rotation and en bloc derotation [26]. Le Naveaux et al. found that ID at the concave side significantly affected coronal correction, while the overall ID was weakly associated to transverse plane correction [2]; they concluded that low-density patterns, with implant mainly placed on the concave side, could result in a comparable simulated deformity correction as higher densities. Delikaris et al. also simulated an additional step of compression/distraction, reporting a strong correlation between ID in the apical region and transverse plane correction, but equivalent results in the coronal and sagittal planes [22].

Early studies on the optimization of personalized surgery planning for AIS patients [27-29], only analyzed a few cases with hybrid (screws/hooks) constructs with fixed implant patterns for each curve and simplified surgical maneuvers (i.e., concave rod rotation) without considering more recent derotation techniques.

The aims of the present comparative computational study are: (i) to investigate how screw pattern and ID affect intraoperative $3 \mathrm{D}$ correction in thoracic scoliosis following concave rod rotation, en bloc derotation and compression/ distraction maneuvers, (ii) to study how correction objectives affect the optimal screw pattern.

Our first hypothesis is that low (ID $\leq 1.4$ screws/level) vs. high (ID $\geq 1.8$ ) density screw patterns would not significantly affect the correction in the three anatomical planes. The second hypothesis is that surgeon's choice of specific correction objectives would greatly influence the choice of the optimal screw pattern.

\section{Materials and methods}

\section{Cases' selection}

10 representative thoracic AIS cases (Lenke 1A) with main thoracic (MT) Cobb angle of $63^{\circ} \pm 6^{\circ}$, a T4-T12 thoracic kyphosis (TK) of $30^{\circ} \pm 20^{\circ}$, an apical vertebral rotation (AVR) of $17^{\circ} \pm 7^{\circ}$ and curve flexibility of $40 \% \pm 17 \%$ were analyzed (Table 1).

\section{Patient-specific biomechanical surgery model}

A personalized computer biomechanical model was built for each case using MD Adams 2018 Multibody Dynamics Simulation Solution (MSC Software, Santa Ana, CA) following an established workflow from calibrated pre-operative biplanar radiographs [2, 21, 22, 24, 26]. Vertebrae were modeled as rigid bodies connected using flexible intervertebral disc and ligament structures, whose stiffness matrix globally was calibrated based on pre-operative clinical side bending radiographs [30].

Homogeneous conditions were analyzed for all curves, rather than simulating the actual surgery; therefore, the simulated posterior fixation included ten vertebrae with uniaxial screws and cobalt-chromium rods with a $5.5 \mathrm{~mm}$ diameter. Rod shapes were reconstructed from available postoperative radiographs. Based on the literature, five alternative screw patterns with a variety of implant densities (independent 
Table 1 Demographic data and geometric indices of the presenting deformities. Flexibility was calculated from side bending radiographs

\begin{tabular}{|c|c|c|c|c|c|c|c|c|c|c|c|}
\hline Patient & $\# 1$ & $\# 2$ & $\# 3$ & $\# 4$ & $\# 5$ & $\# 6$ & $\# 7$ & $\# 8$ & $\# 9$ & $\# 10$ & $\operatorname{Avg} \pm \mathrm{SD}$ \\
\hline Sex & Female & Female & Male & Female & Female & Female & Female & Female & Female & Female & \\
\hline Age (years) & 12 & 18 & 16 & 14 & 17 & 15 & 16 & 17 & 16 & 14 & $16 \pm 2$ \\
\hline Height $(\mathrm{cm})$ & 151 & 156 & 166 & 167 & 156 & 166 & 166 & 166 & 154 & 144 & $159 \pm 2$ \\
\hline Weight (kg) & 39 & 53 & 64 & 57 & 49 & 73 & 70 & 55 & 50 & 42 & $55 \pm 11$ \\
\hline MT Cobb angle $\left(^{\circ}\right)$ & 61 & 57 & 67 & 60 & 69 & 56 & 60 & 63 & 60 & 74 & $63 \pm 6$ \\
\hline $\begin{array}{l}\text { End vertebrae for } \\
\text { Cobb angle evalu- } \\
\text { ation }\end{array}$ & T5-L1 & T5-T12 & T6-L1 & $\mathrm{T} 5-\mathrm{T} 12$ & T7-L2 & T7-L1 & T6-T12 & T5-T12 & T7-L2 & $\mathrm{T} 5-\mathrm{T} 12$ & \\
\hline Curve flexibility (\%) & 25 & 37 & 53 & 74 & 51 & 28 & 23 & 21 & 35 & 50 & $40 \pm 17$ \\
\hline T4-T12 TK $\left(^{\circ}\right)$ & 6 & 40 & 7 & 15 & 34 & 34 & 41 & 34 & 19 & 73 & $30 \pm 20$ \\
\hline $\operatorname{AVR}\left({ }^{\circ}\right)$ & 31 & 14 & 11 & 16 & 16 & 24 & 23 & 8 & 8 & 14 & $17 \pm 7$ \\
\hline Apex & T9 & T9 & T9 & T9 & $\mathrm{T} 10$ & $\mathrm{~T} 10$ & T9 & T9 & $\mathrm{T} 11$ & T9 & \\
\hline
\end{tabular}

Fig. 1 Simulated screw (red dots) patterns and corresponding implant densities (ID). Lowand high-density thresholds of 1.4 and 1.8 were defined according to the MIMO Study Group [18]

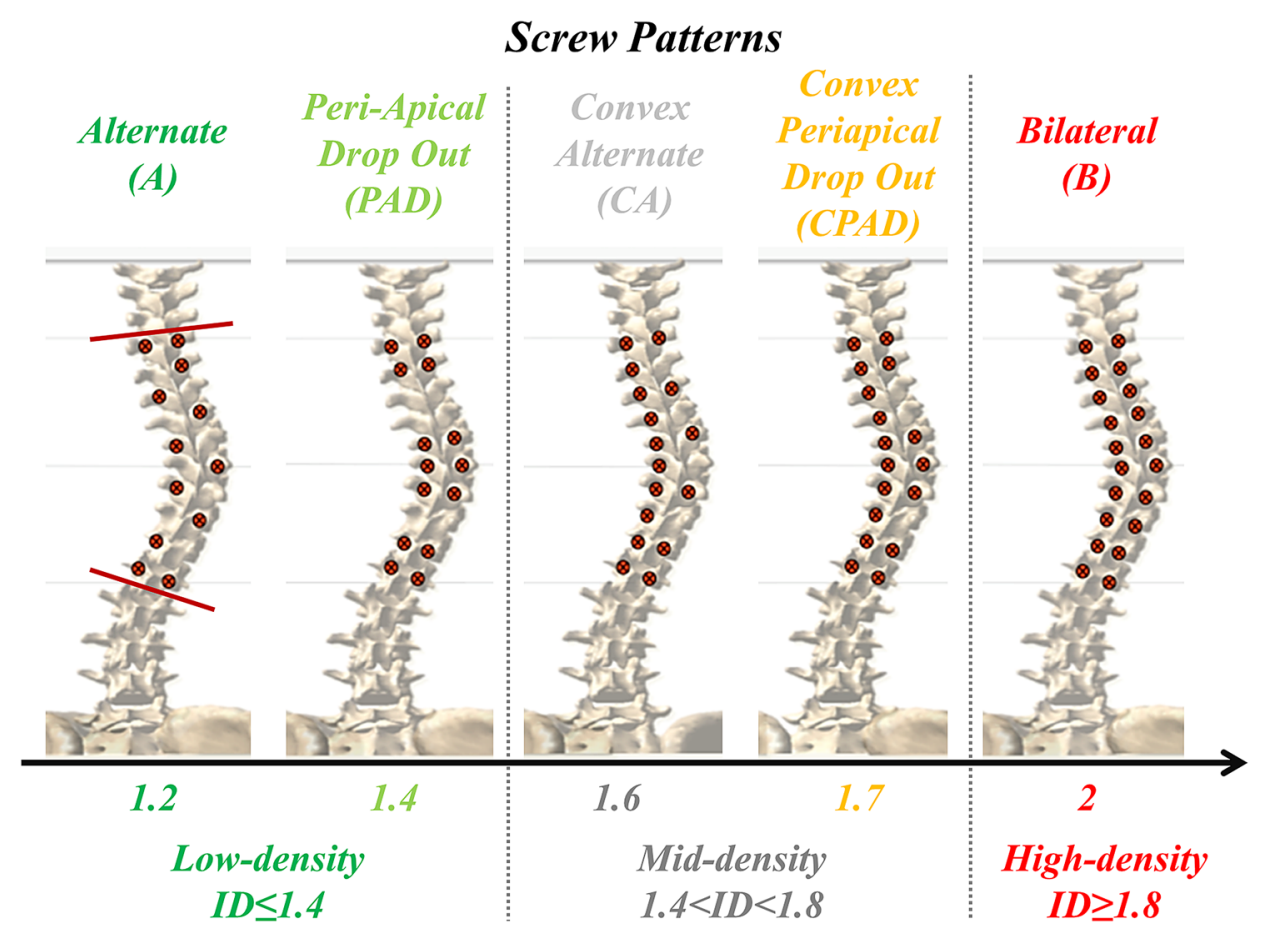

Implant Densities (ID)

variables) were considered for each curve (Fig. 1): alternate ("A", ID = 1.2) $[3,5,10]$, periapical dropout ("PAD", $\mathrm{ID}=1.4$ ) [6], convex alternate ("CA", ID = 1.6) $[5,7,10]$, convex periapical dropout ("CPAD", ID = 1.7) [6] and a full bilateral ("B", ID =2) instrumentation [8,9].

The simulated correction maneuvers were homogeneous for all cases, to isolate the effects of the tested independent variables:

- concave rod positioning and screw engagement,

- concave rod rotation (torque-controlled) [22, 26],

- distal set-screw tightening,
- en bloc derotation (torque-controlled) applied bilaterally on the apical and periapical screws [22,26],

- apical screws tightening,

- convex rod positioning and attachment,

- compression/distraction,

- final screw tightening.

\section{Deformity correction: 3D descriptors and statistical analysis}

To assess the effect of screw pattern and ID (independent variables) on the intraoperative 3D correction of the 
instrumented MT curves, dependent descriptors in the coronal, sagittal and transverse planes were calculated and compared to the corresponding pre-operative condition, as the baseline reference, for each curve: constrained MT Cobb angle, T4-T12 TK and AVR.

After a preliminary Kolmogorov-Smirnov normality check, a two-tailed paired Student $T$-test allowed to compare the simulated 3D descriptors to the reference ones (significance level $p=0.05$ ). The same simulated procedure allowed detecting differences solely due to screw patterns and ID.

To evaluate the sensitivity of the predicted 3D descriptors on screw pattern/ID, their variability was quantified reporting average, standard deviation and overall range (maximum to minimum).

\section{Optimization strategy}

A simple objective function was used to rate the outcome of the virtual surgery weighting the contribution of the predicted 3D descriptors of the scoliotic curve and its residual mobility [27, 28]:

$$
\phi=W_{1} \cdot\left(\frac{M T C \operatorname{Cobb}}{M T C o b b_{0}}\right)^{2}+W_{2} \cdot\left(\frac{T K-T K_{n}}{T K_{0}-T K_{n}}\right)^{2}+W_{3} \cdot\left(\frac{M T A V R}{M T A V R_{0}}\right)^{2}+W_{4} \cdot\left(\frac{N_{\text {Find }}}{N_{0}}\right)^{2}
$$

where the postoperative predicted $M T C o b b$ angle, $T K$ and $M T A V R$ were normalized to the corresponding preoperative measurements (indicated with the underscript "0"). As for the TK, the formula compared the simulated post-op. and the presenting values with the normo-kyphotic range: $T K_{n}=$ $20^{\circ}$ if $T K<20^{\circ}, T K=T K_{n}$ if $20^{\circ} \leq T K \leq 40^{\circ}$ [31, 32], $T K_{n}$ $=40^{\circ}$ if $T K>40^{\circ}$. The mobility term described the postop. number of fused vertebrae ( $\left.N_{\text {Fused }}\right)$ compared to those initially available ( $N_{0}=17$ thoracic and lumbar vertebrae); this term was here assumed constant with $N_{\text {Fused }}=10$ for all the simulated curves and patterns.

Surgeon's preference for one or more of the above-mentioned descriptors, specified by the weighting terms $\left(W_{i}\right)$, was derived from a previous survey on experienced surgeons of the Scoliosis Research Society and Spinal Deformity Study Group (Table 2) [20, 28]. This resulted in 550 possible combinations ( 10 curves, 5 screw patterns/ID, 11 correction objectives).

Ideally, $\phi=1$ would indicate no correction nor residual mobility (total fusion, $N_{\text {Fused }}=N_{0}$ ) compared to pre-op.; $\phi=0$ would indicate a perfect correction with preservation of mobility ( $\left.N_{\text {Fused }}=0\right)$. In general, $\phi<1$ would indicate an improvement in 3D correction, with a reduction of mobility $\left(1 \leq N_{\text {Fused }}<N_{0}\right)$. For a specific set of correction objectives, the minimization of the objective function allowed to determine the optimal screw pattern/ID maximally correcting the deformity in the three planes for of each case, while preserving its mobility.

To evaluate the sensitivity of the output of the objective function on the correction objectives, its variability was quantified for each case, both reporting average \pm standard deviation and the range (maximum-minimum). Moreover, the percentage contribution of each term of the objective function was quantified. The frequency distribution of the optimal screw patterns according to the tested correction objectives was calculated for each case.

\section{Data analysis}

To quantify the sensitivity of the output of the objective function due to the specific curve (i.e., patient), the correction objectives and the screw pattern/ID, a factorial ANOVA was performed in Matlab (significance level $\alpha=0.05$ ).

\section{Results}

\section{D descriptors for deformity correction}

The predicted MT Cobb significantly decreased after instrumentation ( $p<0.05$ ), from 63\% ("A" construct, ID =1.2) up to $67 \%$ ("B", ID =2) of the presenting value. TK did not show significant differences compared to the presenting curve (3-6\% differences, $p>0.81$ ): TK increased of about $12^{\circ}$ for hypo-kyphotic curves (TK $<20^{\circ}$; cases \#1, \#3, \#4), it decreased of $10^{\circ}-22^{\circ}$ for hyper-kyphotic curves (TK $>40$; case \#10), while it did not change for the normo-kyphotic ones. AVR significantly decreased after the simulated instrumentation $(p<0.05)$, improving with ID from 69\% ("A", $\mathrm{ID}=1.2$ ) up to $83 \%$ ("B", $\mathrm{ID}=2$ ) of its presenting value.

No significant difference was detected for any $3 \mathrm{D}$ correction parameter between all tested screw patterns and ID $(p>0.16)$. Screw pattern had a limited effect on simulated MT Cobb, TK and AVR, which varied in average of $3.1^{\circ}$
Table 2 Weights (\%) assigned by 11 surgeons ( $\mathrm{S} 1$ to $\mathrm{S} 11$ ) to each term of the objective function expressing their importance for an optimal 3D correction [20, 28]

\begin{tabular}{|c|c|c|c|c|c|c|c|c|c|c|c|c|}
\hline & & \multicolumn{11}{|c|}{ Correction Objectives } \\
\hline & & S1 & $\mathrm{S} 2$ & S3 & S4 & S5 & S6 & S7 & S8 & S9 & $\mathrm{S} 10$ & $\mathrm{~S} 11$ \\
\hline Coronal plane & $W_{1}$ & 30 & 50 & 30 & 45 & 30 & 20 & 60 & 30 & 25 & 50 & 30 \\
\hline Sagittal plane & $W_{2}$ & 30 & 20 & 30 & 45 & 30 & 50 & 30 & 30 & 10 & 20 & 10 \\
\hline Transverse plane & $W_{3}$ & 20 & 10 & 20 & 10 & 20 & 20 & 10 & 20 & 25 & 20 & 40 \\
\hline Mobility & $W_{4}$ & 20 & 20 & 20 & 0 & 20 & 10 & 0 & 20 & 40 & 10 & 20 \\
\hline
\end{tabular}


$\left( \pm 1.4^{\circ}\right), 2.1^{\circ}\left( \pm 2.0^{\circ}\right)$ and $2.8^{\circ}\left( \pm 1.3^{\circ}\right)$, respectively: the maximum variability of most $3 \mathrm{D}$ descriptors were $\leq 4.2^{\circ}$, with the only exception of case \#6, where it reached $6.6^{\circ}$ and 7.4 $4^{\circ}$, respectively, for Cobb and TK (Table 3).

\section{Variability of the terms of the objective function}

The output of each term of the objective function depended on screw pattern and correction objectives (tested independent variables), with an average value of $0.1( \pm 0.0), 0.4$ $( \pm 0.9), 0.1( \pm 0.1)$, and $0.1( \pm 0.0)$ for the coronal, sagittal, transverse planes and for mobility (Table 4). The maximum variability was 0.1 for the coronal term (case \#7), 3.0 for the sagittal term (case \#7) and 0.2 for the transverse term (case $\# 9)$. $\phi$ had an average overall variability of 0.5 ( \pm 0.9$)$; only cases \#4 and \#7 presented a higher variability (Table 4).

The percentage contribution of each term to the overall output varied from case to case. Considering the average of all screw patterns and correction objectives, the predominant component was the coronal for cases \#1, \#2 and \#8, the sagittal for cases \#4 and \#7, the transverse for cases \#5 and \#9, and mobility for cases \#3, \#6 and \#10 (Table 4).

Table 3 Simulated post-op. values of 3D descriptors (MT Cobb, TK and MT AVR) for each of ten thoracic cases and for each simulated screw pattern

\begin{tabular}{|c|c|c|c|c|c|c|c|c|c|}
\hline \multirow[t]{2}{*}{ Patient } & \multirow[t]{2}{*}{$3 \mathrm{D}$ descriptor $\left({ }^{\circ}\right)$} & \multicolumn{5}{|c|}{ Screw pattern / ID } & \multirow[t]{2}{*}{$\operatorname{Avg} \pm \mathrm{SD}$} & \multirow[t]{2}{*}[\operatorname{min};\operatorname{max}]{} & \multirow[t]{2}{*}{$\max -\min$} \\
\hline & & $\mathrm{A} / 1.2$ & $\mathrm{PAD} / 1.4$ & $\mathrm{CA} / 1.6$ & CPAD /1.7 & $\mathrm{B} / 2$ & & & \\
\hline \multirow[t]{3}{*}{$\# 1$} & MT Cobb & 24 & 24 & 23 & 22 & 22 & $23 \pm 1$ & {$[22 ; 24]$} & 1.9 \\
\hline & TK & 17 & 17 & 18 & 18 & 18 & $18 \pm 0$ & {$[17 ; 18]$} & 1.0 \\
\hline & AVR & 1 & 1 & 1 & 1 & 1 & $1 \pm 0$ & {$[1 ; 1]$} & 0.4 \\
\hline \multirow[t]{3}{*}{$\# 2$} & MT Cobb & 22 & 22 & 19 & 19 & 20 & $20 \pm 2$ & {$[19 ; 22]$} & $3.8^{*}$ \\
\hline & TK & 33 & 31 & 32 & 32 & 32 & $32 \pm 0$ & {$[31 ; 33]$} & 1.3 \\
\hline & AVR & -3 & 1 & -1 & 0 & 0 & $-1 \pm 1$ & {$[-3 ; 1]$} & $3.8^{*}$ \\
\hline \multirow[t]{3}{*}{ \#3 } & MT Cobb & 18 & 17 & 16 & 16 & 15 & $16 \pm 1$ & {$[15 ; 18]$} & 2.7 \\
\hline & TK & 19 & 19 & 20 & 19 & 19 & $19 \pm 0$ & {$[19 ; 20]$} & 1.3 \\
\hline & AVR & -5 & -1 & -2 & -1 & -1 & $-2 \pm 2$ & {$[-5 ;-1]$} & $3.9 *$ \\
\hline \multirow[t]{3}{*}{$\# 4$} & MT Cobb & 19 & 18 & 17 & 17 & 17 & $18 \pm 1$ & {$[17 ; 19]$} & 2.7 \\
\hline & TK & 18 & 15 & 17 & 17 & 17 & $17 \pm 1$ & {$[15 ; 18]$} & $3.2 *$ \\
\hline & AVR & -11 & -10 & -11 & -10 & -11 & $-11 \pm 1$ & {$[-11 ;-10]$} & 1.3 \\
\hline \multirow[t]{3}{*}{ \#5 } & MT Cobb & 28 & 29 & 27 & 26 & 27 & $27 \pm 1$ & {$[26 ; 29]$} & 2.2 \\
\hline & TK & 35 & 37 & 35 & 36 & 36 & $36 \pm 1$ & {$[35 ; 37]$} & 1.8 \\
\hline & AVR & -11 & -13 & -11 & -13 & -12 & $-12 \pm 1$ & {$[-13 ;-11]$} & 1.8 \\
\hline \multirow[t]{3}{*}{ \#6 } & MT Cobb & 24 & 22 & 18 & 18 & 17 & $20 \pm 3$ & {$[17 ; 24]$} & $6.6 * *$ \\
\hline & TK & 39 & 41 & 34 & 36 & 36 & $37 \pm 3$ & {$[34 ; 41]$} & $7.4 * *$ \\
\hline & AVR & 5 & 6 & 7 & 8 & 8 & $7 \pm 1$ & {$[5 ; 8]$} & $3.5^{*}$ \\
\hline \multirow[t]{3}{*}{$\# 7$} & MT Cobb & 27 & 25 & 25 & 25 & 25 & $26 \pm 1$ & {$[25 ; 27]$} & 2.5 \\
\hline & TK & 43 & 43 & 42 & 42 & 42 & $42 \pm 1$ & {$[42 ; 43]$} & 1.3 \\
\hline & AVR & -14 & -11 & -11 & -11 & -11 & $-12 \pm 1$ & {$[-14 ;-11]$} & 2.7 \\
\hline \multirow[t]{3}{*}{ \#8 } & MT Cobb & 27 & 25 & 24 & 24 & 24 & $25 \pm 1$ & {$[24 ; 27]$} & $3.2 *$ \\
\hline & TK & 35 & 35 & 34 & 34 & 34 & $35 \pm 0$ & {$[34 ; 35]$} & 1.1 \\
\hline & AVR & -1 & 3 & 2 & 3 & 3 & $2 \pm 2$ & {$[-1 ; 3]$} & $4.2 *$ \\
\hline \multirow[t]{3}{*}{ \#9 } & MT Cobb & 23 & 21 & 20 & 19 & 20 & $20 \pm 1$ & {$[19 ; 23]$} & $3.3 *$ \\
\hline & TK & 30 & 31 & 30 & 30 & 30 & $30 \pm 0$ & {$[30 ; 31]$} & 0.9 \\
\hline & AVR & -6 & -5 & -4 & -4 & -4 & $-5 \pm 1$ & {$[-6 ;-4]$} & 2.5 \\
\hline \multirow[t]{3}{*}{ \#10 } & MT Cobb & 19 & 18 & 18 & 18 & 21 & $19 \pm 1$ & {$[18 ; 21]$} & 2.2 \\
\hline & TK & 51 & 51 & 50 & 50 & 50 & $50 \pm 1$ & {$[50 ; 51]$} & 1.9 \\
\hline & AVR & -6 & -2 & -3 & -2 & -2 & $-3 \pm 2$ & {$[-6 ;-2]$} & $3.8^{*}$ \\
\hline
\end{tabular}

Their variability is expressed as average (Avg) \pm standard deviation(SD) with range (Max-min). The double asterisk ("**") indicates the maximum variability of 3D descriptors, while a single asterisk ("*") indicates when the variability is greater than the average value calculated over all ten cases 
Table 4 Variability of each term of the objective function, expressed as average \pm SD with range (max-min), due to a variation in correction objectives and screw pattern/ ID for each curve (55 possible combinations: 5 screw patterns/ implant densities and 11 correction objectives). The percentage contribution to the overall output of the objective function is also reported

\begin{tabular}{|c|c|c|c|c|c|c|}
\hline \multirow[t]{2}{*}{ Patient } & \multirow[t]{2}{*}{$\begin{array}{l}\text { Terms of the objec- } \\
\text { tive function }\end{array}$} & \multirow[t]{2}{*}{$\operatorname{Avg} \pm \mathrm{SD}$} & \multirow[t]{2}{*}[\operatorname{min};\operatorname{max}]{} & \multirow[t]{2}{*}{ Max-min } & \multicolumn{2}{|c|}{$\begin{array}{l}\text { Contribution to the objec- } \\
\text { tive function }(\%)\end{array}$} \\
\hline & & & & & $\operatorname{Avg} \pm \mathrm{SD}$ & {$[\min ; \max ]$} \\
\hline \multirow[t]{4}{*}{$\# 1$} & Coronal plane & $0.1 \pm 0.0$ & {$[0.0 ; 0.1]$} & $0.2 *$ & $47 \pm 22$ & {$[20 ; 90]$} \\
\hline & Sagittal plane & $0.0 \pm 0.0$ & {$[0.0 ; 0.0]$} & 0.0 & $8 \pm 6$ & {$[2 ; 19]$} \\
\hline & Transverse plane & $0.0 \pm 0.0$ & {$[0.0 ; 0.0]$} & 0.0 & $0 \pm 0$ & {$[0 ; 0]$} \\
\hline & Mobility & $0.1 \pm 0.0$ & {$[0.0 ; 0.1]$} & 0.1 & $44 \pm 25$ & {$[0 ; 78]$} \\
\hline \multirow[t]{4}{*}{ \#2 } & Coronal plane & $0.1 \pm 0.0$ & {$[0.0 ; 0.1]$} & $0.1^{*}$ & $49 \pm 27$ & {$[19 ; 99]$} \\
\hline & Sagittal plane & $0.0 \pm 0.0$ & {$[0.0 ; 0.0]$} & 0.0 & $0 \pm 0$ & {$[0 ; 0]$} \\
\hline & Transverse plane & $0.0 \pm 0.0$ & {$[0.0 ; 0.0]$} & 0.0 & $2 \pm 1$ & {$[1 ; 4]$} \\
\hline & Mobility & $0.1 \pm 0.0$ & {$[0.0 ; 0.1]$} & 0.1 & $44 \pm 25$ & {$[0 ; 78]$} \\
\hline \multirow[t]{4}{*}{ \#3 } & Coronal plane & $0.0 \pm 0.0$ & {$[0.0 ; 0.0]$} & 0.0 & $33 \pm 27$ & {$[9 ; 86]$} \\
\hline & Sagittal plane & $0.0 \pm 0.0$ & {$[0.0 ; 0.0]$} & 0.0 & $1 \pm 1$ & {$[0 ; 4]$} \\
\hline & Transverse plane & $0.0 \pm 0.0$ & {$[0.0 ; 0.1]$} & 0.1 & $11 \pm 4$ & {$[5 ; 18]$} \\
\hline & Mobility & $0.1 \pm 0.0$ & {$[0.0 ; 0.1]$} & 0.1 & $55 \pm 29$ & {$[0 ; 84]$} \\
\hline \multirow[t]{4}{*}{$\# 4$} & Coronal plane & $0.0 \pm 0.0$ & {$[0.0 ; 0.1]$} & 0.1 & $10 \pm 5$ & {$[4 ; 20]$} \\
\hline & Sagittal plane & $0.2 \pm 0.2$ & {$[0.0 ; 0.7]$} & $0.7 *$ & $47 \pm 18$ & {$[17 ; 75]$} \\
\hline & Transverse plane & $0.1 \pm 0.0$ & {$[0.0 ; 0.2]$} & $0.2 *$ & $26 \pm 11$ & {$[13 ; 53]$} \\
\hline & Mobility & $0.1 \pm 0.0$ & {$[0.0 ; 0.1]$} & 0.1 & $17 \pm 12$ & {$[0 ; 43]$} \\
\hline \multirow[t]{4}{*}{ \#5 } & Coronal plane & $0.1 \pm 0.0$ & {$[0.0 ; 0.1]$} & $0.1^{*}$ & $29 \pm 17$ & {$[12 ; 63]$} \\
\hline & Sagittal plane & $0.0 \pm 0.0$ & {$[0.0 ; 0.0]$} & 0.0 & $0 \pm 0$ & {$[0 ; 0]$} \\
\hline & Transverse plane & $0.1 \pm 0.1$ & {$[0.1 ; 0.3]$} & $0.2 *$ & $\mathbf{4 8} \pm 11$ & {$[27 ; 66]$} \\
\hline & Mobility & $0.1 \pm 0.0$ & {$[0.0 ; 0.1]$} & 0.1 & $23 \pm 14$ & {$[0 ; 44]$} \\
\hline \multirow[t]{4}{*}{ \#6 } & Coronal plane & $0.1 \pm 0.0$ & {$[0.0 ; 0.1]$} & $0.1^{*}$ & $42 \pm 24$ & {$[16 ; 88]$} \\
\hline & Sagittal plane & $0.0 \pm 0.0$ & {$[0.0 ; 0.0]$} & 0.0 & $1 \pm 1$ & {$[0 ; 4]$} \\
\hline & Transverse plane & $0.0 \pm 0.0$ & {$[0.0 ; 0.1]$} & 0.0 & $14 \pm 5$ & {$[6 ; 25]$} \\
\hline & Mobility & $0.1 \pm 0.0$ & {$[0.0 ; 0.1]$} & 0.1 & $42 \pm 23$ & {$[0 ; 72]$} \\
\hline \multirow[t]{4}{*}{ \#7 } & Coronal plane & $0.1 \pm 0.0$ & {$[0.0 ; 0.1]$} & $0.1 * *$ & $6 \pm 33$ & {$[2 ; 10]$} \\
\hline & Sagittal plane & $1.1 \pm 0.7$ & {$[0.2 ; 3.2]$} & $3.0 * *$ & $83 \pm 11$ & {$[61 ; 94]$} \\
\hline & Transverse plane & $0.1 \pm 0.0$ & {$[0.0 ; 0.1]$} & $0.1 *$ & $5 \pm 4$ & {$[1 ; 16]$} \\
\hline & Mobility & $0.1 \pm 0.0$ & {$[0.0 ; 0.1]$} & 0.1 & $6 \pm 6$ & {$[0 ; 22]$} \\
\hline \multirow[t]{4}{*}{ \#8 } & Coronal plane & $0.1 \pm 0.0$ & {$[0.0 ; 0.1]$} & $0.1^{*}$ & $46 \pm 24$ & {$[19 ; 91]$} \\
\hline & Sagittal plane & $0.0 \pm 0.0$ & {$[0.0 ; 0.0]$} & 0.0 & $0 \pm 0$ & {$[0 ; 0]$} \\
\hline & Transverse plane & $0.0 \pm 0.0$ & {$[0.0 ; 0.1]$} & 0.1 & $14 \pm 5$ & {$[6 ; 25]$} \\
\hline & Mobility & $0.1 \pm 0.0$ & {$[0.0 ; 0.1]$} & 0.1 & $39 \pm 22$ & {$[0 ; 69]$} \\
\hline \multirow[t]{4}{*}{ \#9 } & Coronal plane & $0.0 \pm 0.0$ & {$[0.0 ; 0.1]$} & 0.1 & $29 \pm 18$ & {$[11 ; 65]$} \\
\hline & Sagittal plane & $0.0 \pm 0.0$ & {$[0.0 ; 0.0]$} & 0.0 & $0 \pm 0$ & {$[0 ; 0]$} \\
\hline & Transverse plane & $0.1 \pm 0.1$ & {$[0.0 ; 0.3]$} & $0.2 * *$ & $42 \pm 10$ & {$[22 ; 59]$} \\
\hline & Mobility & $0.1 \pm 0.0$ & {$[0.0 ; 0.1]$} & 0.1 & $30 \pm 17$ & {$[0 ; 53]$} \\
\hline \multirow[t]{4}{*}{$\# 10$} & Coronal plane & $0.0 \pm 0.0$ & {$[0.0 ; 0.1]$} & 0.0 & $22 \pm 13$ & {$[9 ; 53]$} \\
\hline & Sagittal plane & $0.0 \pm 0.0$ & {$[0.0 ; 0.1]$} & 0.1 & $26 \pm 16$ & {$[6 ; 56]$} \\
\hline & Transverse plane & $0.0 \pm 0.0$ & {$[0.0 ; 0.1]$} & 0.1 & $9 \pm 3$ & {$[4 ; 18]$} \\
\hline & Mobility & $0.1 \pm 0.0$ & {$[0.0 ; 0.1]$} & 0.1 & $\mathbf{4 3} \pm 24$ & {$[0 ; 78]$} \\
\hline
\end{tabular}

The double asterisk ("**") indicates the maximum variability of each term of the objective function, while a single asterisk ("*") indicates when the variability is greater than the average value calculated over all ten curves. The percentage contribution to the overall output of the objective function is expressed as Avg \pm SD with range 
Table 5 Overall variability of the objective function and corresponding optimal screw patterns for each curve. When the optimum is unique, the corresponding screw pattern is bolded; when more than one screw pattern resulted to be optimal, their frequency distribution over the 11 tested correction objectives is reported as a percentage

\begin{tabular}{lllll}
\hline Patient & Avg \pm SD & {$[\min ; \max ]$} & Max-min $\begin{array}{l}\text { Optimal screw } \\
\text { patterns }\end{array}$ \\
\hline$\# 1$ & $0.1 \pm 0.0$ & {$[0.1 ; 0.2]$} & 0.1 & $\begin{array}{c}\text { CA: } 55 \% \\
\text { CPAD: } 45 \%\end{array}$ \\
& & & & \\
$\# 2$ & $0.1 \pm 0.0$ & {$[0.1 ; 0.2]$} & 0.1 & CPAD \\
$\# 3$ & $0.1 \pm 0.0$ & {$[0.0 ; 0.2]$} & 0.2 & B \\
$\# 4$ & $0.3 \pm 0.1$ & {$[0.2 ; 0.8]$} & $0.7 * *$ & A: $73 \%$ CPAD: \\
& & & & $27 \%$ \\
$\# 5$ & & & & \\
$\# 6$ & $0.2 \pm 0.1$ & {$[0.1 ; 0.4]$} & 0.3 & CA \\
& $0.1 \pm 0.0$ & {$[0.1 ; 0.2]$} & 0.1 & CA: 73\%; B: \\
& & & & $27 \%$ \\
$\# 7$ & $1.3 \pm 0.7$ & {$[0.4 ; 3.3]$} & 2.9 & CA \\
$\# 8$ & $0.1 \pm 0.0$ & {$[0.1 ; 0.2]$} & $0.1 * *$ & A: 64\%; CPAD: \\
& & & & $27 \% ;$ CA: $9 \%$ \\
$\# 9$ & $0.2 \pm 0.1$ & {$[0.1 ; 0.4]$} & 0.3 & CPAD \\
$\# 10$ & $0.1 \pm 0.0$ & {$[0.1 ; 0.2]$} & 0.1 & CPAD: \\
& & & & $91 \% ;$ CA: 9\% \\
\hline
\end{tabular}

The double asterisk ("**") indicates the maximum variability of objective function output, while a single asterisk ("*") indicates when the variability is greater than the average value calculated over all ten cases

\section{Optimal screw pattern (and ID)—General trends}

Among all the curves and the tested correction objectives, "CPAD" pattern was optimal in $39 \%$ of analyzed scenarios (43/110), followed by "CA" in 35\% (38/110), "A" in $14 \%$ $(15 / 110)$ and "B" in $13 \%$ (14/110). When grouping by ID, low-density patterns resulted optimal in $14 \%$ of analyzed scenarios (or 15/110), mid-density patterns in $74 \%$ (or $81 / 110)$ and high-density in 13\% (14/110).

\section{Optimal screw pattern (and ID)_-Specific trends}

The optimal pattern was unique for $50 \%$ of the patients. One specific mid-density pattern was optimal in three cases: "CA" for cases \#5 and \#7, "CPAD" for cases \#2 and \#9. The "B" high-density pattern was optimal for case \#3. Multiple optimal patterns were found for the remaining patients, depending on the tested correction objectives (Table 5). The optimal pattern was within the mid-density range for cases $\# 1$ and \#10. The optimum could span from low- to middensity patterns both for cases \#4 ("A": 8/11, "CPAD": 3/11) and \#8 ("A": 7/11, "CPAD": 3/11, "CA": 1/11). In case \#6, the optimal pattern could span from mid- ("CA": 8/11) to high-density ("B": 3/11).

\section{Data analysis}

The ANOVA indicated that the output of the objective function was significantly affected by the specific curve $(97 \%$, $p<0.05$ ), rather than by the correction objectives and the screw pattern/ID. When repeating the ANOVA for each term of the objective function, the specific curve could explain $36 \%(p<0.05), 96 \%(p<0.05)$ and $84 \%(p<0.05)$ of the variability of the coronal, the sagittal and transverse terms, respectively; while the correction objectives could explain $56 \%$ ( $p<0.05$, higher than patient effect) of the coronal term and $7 \%(p<0.05)$ of the transverse one; and the screw pattern/ID only explained an $8 \%(p<0.05)$ of the coronal term.

\section{Discussion}

The present biomechanical study systematically compared five similar screw patterns and IDs (independent parameters), widely accepted among experienced spine surgeons [3, 5-11], while controlling the preparation technique (no osteotomies or ligament release), the instrumented levels and correction maneuvers (confounding parameters here constant). Conversely, traditional clinical studies included inhomogeneous hybrid patterns, various implant densities, fused levels and correction techniques [11, 15-17].

The first hypothesis that low- and high-density screw patterns would provide equivalent 3D correction was confirmed, as we did not find statistically significant differences on coronal, sagittal and transverse plane correction. This generally agrees with previous biomechanical studies on thoracic AIS curves describing concave rod rotation, en bloc derotation and eventual compression/distraction [2, 21, 22, 26]. Compared to our study (ID range 1.2-2; 10 fused levels), they considered also lower, but today less common, IDs (range 0.73-2; fused levels 10-11) [2, 26], reporting significant effects/correlations with ID only in few specific cases.

Our results are consistent with the MIMO prospective RCT on Lenke 1A curves, reporting equivalent coronal correction of MT Cobb (range 63\%-67\%) and unchanged TK using low-/high-densities [18], however, correction maneuvers and implant patterns were not documented. When considering concave rod rotation and eventual compression/distraction, published retrospective studies reported equivalent coronal $(63 \%-76 \%)$ [4, 5, 10, 33-35], sagittal (TK change $\left.-6^{\circ}-14^{\circ}\right)[4,5,10,21,33-35]$ and transverse plane correction (AVR change: $1^{\circ}$ ) [33] mixing various patterns and arbitrary definitions of low/high IDs. When considering our same set of maneuvers, an equivalent coronal correction (80\%-84\%) was again reported for relatively low IDs (1.1 vs. 1.3)[36]. Although we did not, they reported unsatisfactory sagittal restoration with lower IDs and small $(5.5 \mathrm{~mm})$ rod diameters, while a better TK with higher ID and bigger 
size $(6.35 \mathrm{~mm})$ rods [36]. Our same qualitative trend on TK, with a slight decrease in hyper-kyphotic curves and significant increase in hypo-kyphotic ones, have been reported $[4,21]$. Although variations in the surgical technique and implants (i.e., rods contour/size) may explain some relative differences among studies [2], our analysis confirmed that screw pattern and ID play a minor contribution on correction, when pooling the correction maneuvers, the number of fused levels and using CoCr $5.5 \mathrm{~mm}$ rods. Based on preliminary simulations [41], we also ensured that the effect of rod diameter on 3D correction was even lower than the effect of screw pattern and ID herein presented. Considering the impact of using high-density constructs on increased surgery time, blood loss, complications and costs [14, 15, 34], using fewer implants to achieve adequate correction could be considered.

This paper provides an important novel contribution in that it identifies the optimal screw patterns and implant densities linked to specific correction objectives. Previous studies focused only on hybrid constructs, fixed implant pattern for each curve without modern derotation techniques [27-29]. The current study demonstrates that the choice of the same correction objectives would not result in the same optimal screw pattern in a representative cohort of ten thoracic curves, confirming our second hypothesis and supporting three ideas. Firstly, each curve is unique due to a combination of patient-specific variables (i.e., curve flexibility/extension, apex location), stressing on the need for a personalized approach to support the decision-making process for pre-operative surgical planning. Unfortunately, when comparing patients' characteristics (i.e., curve flexibility, pre-op. Cobb, pre-op. thoracic kyphosis, pre-op. apical vertebral rotations, \% coronal correction, variation in thoracic kyphosis, \% transverse correction) with the characteristics of the screw patterns and IDs identified as optimal, we did not notice anything relevant. The ANOVA indicated that the objective function was significantly more affected by the specific curve (i.e., patient) rather than by the correction objectives and the screw patterns or IDs; therefore, we suspect that a higher number of curves would be needed to possibly assess how patients' characteristics are associated with the optimal instrumentation strategy. As second, the identification of the optimal screw pattern/ID depends on subjective correction objectives with multiple optimal pattern being equally justified for the same curve. As the spectrum of surgical choices is wide $[11,12,16,17,19,20]$, only a systematic individualized approach like the one here proposed could help rationalizing the decision-making process and reduce its variability. As third, correction objectives have higher impact than the tested independent parameters on the optimization result. For instance, a too low weight factor may hide the effect of a clinically relevant $\left(>5^{\circ}\right)$ variation of a specific deformity indices in a specific plane; vice versa, a too high weight factor may overweight the effect of a not clinically relevant $\left(<5^{\circ}\right)$ variation in other plane. To avoid these situations and preserve useful information, the weight factors' range could be limited depending on the variability of each corresponding 3D descriptor. This is expected to be clearer as multiple independent parameters (e.g., rods tracing and stiffness, the number of fused vertebrae, correction maneuvers, spine flexibility...), which are expected to have a much higher impact on 3D correction than screw pattern and ID alone, are included in the modelling.

The tested correction objectives were extracted from a survey at a period where surgeons were rating more the coronal plane (Table 2) [20, 28]. Nowadays, bi-planar lowdose radiography and transverse plane maneuvers are more established, while the attention on sagittal plane and balance is increasing [37]; therefore, correction perspectives could be different today.

Assumptions in the modeling may set some limitations on the results of this study. To limit the number of tested independent variables, the same number of fused levels, fixed screw patterns/ID and correction techniques were simulated for all cases. While, in reality, surgeons may adjust the surgical technique depending on the available anchoring points, curve flexibility and span a wider range of possibilities [2, $11,26]$. Additional factors, such the anchor type [27, 29, 38], rod characteristics (i.e., contour, material, diameter) [27, 39, 40], other surgical maneuvers (i.e., segmental derotation, in situ rod bending) [21], might affect the correction in the three anatomical planes. Moreover, changing the number of fused levels (upper/lower instrumented vertebrae) [27-29] would impact spine mobility in the objective function. Only a more general simulation study accounting for all the desired instrumentation parameters and their interactions, could establish how they would ensure an optimal correction [41].

The objective function here considered was limited to one key metric per plane describing the intraoperative condition, but it may be further expanded to include other spine descriptor, as well as other non-geometrical parameters (i.e., loads at implants/bone interface, loads on pedicle screws and spinal rods, loads on the anterior spine), which have already been reported in dedicated studies [2, 22, 25, 26]. The presented approach has the potential to be used in future complementary study to evaluate other research questions, such as the risks of failure due to postoperative functional loads arising during the everyday life activities. The developed optimization approach could be applied to any other Lenke curve type. Although these aspects would merit further investigation, the effect of our study assumptions on the final conclusions could be, nevertheless, considered as 
limited, given its comparative nature and its focus on screw pattern and ID.

To conclude, this biomechanical comparative study demonstrates that screw patterns with low (ID $\leq 1.4$ screws/level) and high (ID $\geq 1.8$ ) implant densities provide equivalent intraoperative correction in the three anatomical planes in a representative cohort of ten thoracic AIS curves. Moreover, the identification of the optimal screw pattern for every curve depends on surgeons' preference regarding specific correction objectives.

The proposed patient-specific approach represents a promising tool to assess and optimize the surgery planning of complex spinal deformities including surgeon-dependent correction objectives.

Author contributions The enlisted authors contributed according to the listed criteria: LLB, ANL, CEA: Made substantial contributions to the conception or design of the work; or the acquisition, analysis, or interpretation of data; or the creation of new software used in the work. LLB, ANL, CEA: Drafted the work or revised it critically for important intellectual content. LLB, ANL, CEA: Approved the version to be published. LLB, ANL, CEA: Agree to be accountable for all aspects of the work in ensuring that questions related to the accuracy or integrity of any part of the work are appropriately investigated and resolved.

Funding Open Access funding provided by Politecnico di Milano LLB: Canada First Research Excellence Fund through TransMedTech Institute Postdoctoral Fellowship. CEA: Natural Sciences and Engineering Research Council of Canada (NSERC)-Medtronic Industrial Research Chair in Spine Biomechanics, Canada Research Chair in Orthopedic Engineering.

\section{Compliance with ethical standards}

Conflict of interest ANL: funds apart from this study from Globus, Medtronic, Zimmer, and Orthopediatrics.

Ethical approval Approval was obtained from the ethics committee of the involved institutions.

Informed consent Informed consent was obtained from all participants included in the study.

Open Access This article is licensed under a Creative Commons Attribution 4.0 International License, which permits use, sharing, adaptation, distribution and reproduction in any medium or format, as long as you give appropriate credit to the original author(s) and the source, provide a link to the Creative Commons licence, and indicate if changes were made. The images or other third party material in this article are included in the article's Creative Commons licence, unless indicated otherwise in a credit line to the material. If material is not included in the article's Creative Commons licence and your intended use is not permitted by statutory regulation or exceeds the permitted use, you will need to obtain permission directly from the copyright holder. To view a copy of this licence, visit http://creativecommons.org/licenses/by/4.0/.

\section{References}

1. Lenke LG, Kuklo TR, Ondra S et al (2008) Rationale behind the current state-of-the-art treatment of scoliosis (in the pedicle screw era). Spine 33(10):1051-1054. https://doi.org/10.1097/ BRS.0b013e31816f2865

2. Le Navéaux F, Larson AN, Labelle H, et al (2016) How does implant distribution affect 3D correction and bone-screw forces in thoracic adolescent idiopathic scoliosis spinal instrumentation? Clin Biomech (Bristol. Avon) 39:25-31. doi: https://doi. org/10.1016/j.clinbiomech.2016.09.002.

3. Kemppainen JW, Morscher MA, Gothard MD et al (2016) Evaluation of limited screw density pedicle screw constructs in posterior fusions for adolescent idiopathic scoliosis. Spine Deform 4(1):33-39. https://doi.org/10.1016/j.jspd.2015.07.010

4. Le Navéaux F, Aubin CÉ, Larson AN, et al (2015) Implant distribution in surgically instrumented Lenke 1 adolescent idiopathic scoliosis: does it affect curve correction? Spine (Phila Pa 1976) 40(7):462-8. doi: https://doi.org/10.1097/BRS.0000000000000793.

5. Gotfryd AO, Avanzi O (2013) Randomized clinical study on surgical techniques with different pedicle screw densities in the treatment of adolescent idiopathic scoliosis types Lenke 1A and 1B. Spine Deform 1(4):272-279. https://doi.org/10.1016/j. jspd.2013.05.004

6. Bharucha NJ, Lonner BS, Auerbach JD et al (2013) Low-density versus high-density thoracic pedicle screw constructs in adolescent idiopathic scoliosis: do more screws lead to a better outcome? Spine J 13:375-381. https://doi.org/10.1016/j.spine e.2012.05.029

7. Hwang CJ, Lee CK, Chang BS et al (2011) Minimum 5-year follow-up results of skipped pedicle screw fixation for flexible idiopathic scoliosis. J Neurosurg Spine 15(2):146-150. https:// doi.org/10.3171/2011.4.SPINE10321

8. Quan GM, Gibson MJ (2010) Correction of main thoracic adolescent idiopathic scoliosis using pedicle screw instrumentation: does higher implant density improve correction? Spine (Phila Pa 1976) 35(5):562-7. doi: https://doi.org/10.1097/BRS.0b013e3181 b4af34.

9. Clements DH, Betz RR, Newton PO, et al (2009) Correlation of scoliosis curve correction with the number and type of fixation anchors. Spine (Phila Pa 1976) 34(20):2147-50. doi: https://doi. org/10.1097/BRS.0b013e3181adb35d.

10. Li M, Shen Y, Fang X et al (2009) Coronal and sagittal plane correction in patients with Lenke 1 adolescent idiopathic scoliosis: a comparison of consecutive versus interval pedicle screw placement. J Spinal Disord Tech 22(4):251-256. https://doi. org/10.1097/BSD.0b013e3181884940

11. Le Navéaux F, Larson AN, Labelle H et al (2018) Significant variability in surgeons' preferred correction maneuvers and instrumentation strategies when planning adolescent idiopathic scoliosis surgery. Scoliosis Spinal Disord 13:21. https://doi.org/10.1186/ s13013-018-0169-8

12. Aubin CE, Larson AN, Le Navéaux F, et al (2012) Active SRS members demonstrate huge variation in implant density when planning routine adolescent idiopathic scoliosis constructs. Scoliosis Research Society 47th Annual Meeting and Course. Chicago. USA. p. 140.

13. Robitaille M, Aubin CE, Labelle H (2007) Intra and interobserver variability of preoperative planning for surgical instrumentation in adolescent idiopathic scoliosis. Eur Spine J 16(10):1604-1614. https://doi.org/10.1007/s00586-007-0431-x

14. Larson AN, Polly DW, Ackerman SJ et al (2016) What would be the annual cost savings if fewer screws were used in adolescent idiopathic scoliosis treatment in the US? J Neurosurg Spine 24(1):116-123. https://doi.org/10.3171/2015.4.SPINE131119 
15. Larson AN, Lonner BS (2015) Comparative effectiveness of implant density. Seminars in Spine Surgery 27(1):45-51. https:// doi.org/10.1053/j.semss.2015.01.010

16. Larson AN, Polly DW, Diamond B, et al (2014) Does higher anchor density result in increased curve correction and improved clinical outcomes in adolescent idiopathic scoliosis? Spine (Phila Pa 1976) 39(7):571-8. doi: https://doi.org/10.1097/BRS.00000 00000000204.

17. Larson AN, Aubin CE, Polly DW et al (2013) Are more screws better? A systematic review of anchor density and curve correction in adolescent idiopathic scoliosis. Spine Deform 1(4):237247. https://doi.org/10.1016/j.jspd.2013.05.009

18. Larson NA, Polly DW, Sponseller PD, et al (2019) Prospective randomized controlled trial of implant density in AIS: results of the Minimize Implants Maximize Outcomes Trial. Paper \#78. Annual Meeting of the Scoliosis Research Society, September 20, Montreal, Canada.

19. Aubin CE, Labelle H, Ciolofan OC (2007) Variability of spinal instrumentation configurations in adolescent idiopathic scoliosis. Eur Spine J 16(1):57-64. https://doi.org/10.1007/s0058 6-006-0063-6

20. Majdouline Y, Aubin CE, Robitaille M et al (2007) Scoliosis correction objectives in adolescent idiopathic scoliosis. J Pediatr Orthop 27(7):775-781. https://doi.org/10.1097/BPO.0b013e3181 $5588 \mathrm{~d} 8$

21. Boyer L (2017) Analyse biomécanique de techniques de dérotation vertébrale pour la correction 3D de la scoliose lors de la chirurgie d'instrumentation postérieure du rachis. Doctoral thesis. December. École Polytechnique de Montréal.

22. Delikaris A, Wang X, Boyer L, et al (2018) Implant density at the apex is more important than overall implant density for 3D correction in thoracic adolescent idiopathic scoliosis using rod derotation and en bloc vertebral derotation technique. Spine (Phila Pa 1976) 43(11):E639-47. doi: https://doi.org/10.1097/BRS.00000 00000002465.

23. Galbusera F, Bassani T, La Barbera L, et al (2015) Planning the surgical correction of spinal deformities: toward the identification of the biomechanical principles by means of numerical simulation. Front Bioeng Biotechnol 3(178). doi: https://doi.org/10.3389/ fbioe.2015.00178

24. Aubin CE, Labelle H, Chevrefils C, et al (2008) Preoperative planning simulator for spinal deformity surgeries. Spine (Phila Pa 1976) 33(20):2143-52. doi: https://doi.org/10.1097/BRS.0b013 e31817bd89f.

25. Wang X, Aubin CE, Robitaille I et al (2012) Biomechanical comparison of alternative densities of pedicle screws for the treatment of adolescent idiopathic scoliosis. Eur Spine J 21(6):1082-1090. https://doi.org/10.1007/s00586-011-2089-7

26. Martino J, Aubin CE, Labelle H, et al (2013) Biomechanical analysis of vertebral derotation techniques for the surgical correction of thoracic scoliosis. A numerical study through case simulations and a sensitivity analysis. Spine (Phila Pa 1976) 38(2):E73-83. doi: https://doi.org/10.1097/BRS.0b013e31827a641e.

27. Majdouline Y, Aubin CE, Sangole A et al (2009) Computer simulation for the optimization of instrumentation strategies in adolescent idiopathic scoliosis. Med Biol Eng Comput 47(11):11431154. https://doi.org/10.1007/s11517-009-0509-1

28. Majdouline Y, Aubin CE, Wang X et al (2012) Preoperative assessment and evaluation of instrumentation strategies for the treatment of adolescent idiopathic scoliosis: computer simulation and optimization. Scoliosis 7(1):21. https://doi. org/10.1186/1748-7161-7-21

29. Robitaille M, Aubin CE, Labelle H (2009) Effects of alternative instrumentation strategies in adolescent idiopathic scoliosis: a biomechanical analysis. J Orthop Res 27(1):104-113. https://doi. org/10.1002/jor.20654

30. Petit Y, Aubin CE, Labelle H (2004) Patient-specific mechanical properties of a flexible multi-body model of the scoliotic spine. Med Biol Eng Comput 42(1):55-60. https://doi.org/10.1007/bf023 51011

31. Sucato DJ, Agrawal S, O'Brien MF, et al. Richards SB. Lenke L (2008) Restoration of thoracic kyphosis after operative treatment of adolescent idiopathic scoliosis: a multicenter comparison of three surgical approaches. Spine (Phila Pa 1976) 33(24):2630-6. doi: https://doi.org/10.1097/BRS.0b013e3181880498.

32. de Jonge T, Dubousset JF, Illés T (2002) Sagittal plane correction in idiopathic scoliosis. Spine (Phila Pa 1976) 27(7):754-60.

33. Yeh YC, Niu CC, Chen LH et al (2019) The correlations between the anchor density and the curve correction of adolescent idiopathic scoliosis surgery. BMC Musculoskelet Disord 20(1):497. https://doi.org/10.1186/s12891-019-2844-1

34. Shen M, Jiang H, Luo M et al (2017) Comparison of low density and high density pedicle screw instrumentation in Lenke 1 adolescent idiopathic scoliosis. BMC Musculoskelet Disord 18(1):336. https://doi.org/10.1186/s12891-017-1695-x

35. Luo M, Shen M, Wang W et al (2017) Comparison of consecutive, interval, and skipped pedicle screw techniques in moderate lenke type 1 adolescent idiopathic scoliosis. World Neurosurg 98:563-570. https://doi.org/10.1016/j.wneu.2016.11.064

36. Liu H, Li Z, Li S et al (2015) Main thoracic curve adolescent idiopathic scoliosis: association of higher rod stiffness and concaveside pedicle screw density with improvement in sagittal thoracic kyphosis restoration. J Neurosurg Spine 22(3):259-266. https:// doi.org/10.3171/2014.10.SPINE1496

37. Abelin-Genevois K, Sassi D, Verdun S et al (2018) Sagittal classification in adolescent idiopathic scoliosis: original description and therapeutic implications. Eur Spine J 27(9):2192-2202. https ://doi.org/10.1007/s00586-018-5613-1

38. Wang X, Aubin CE, Coleman J, Rawlinson J (2017) Correction capability in the 3 anatomic planes of different pedicle screw designs in scoliosis instrumentation. Clin Spine Surg 30(4):E323E330. https://doi.org/10.1097/BSD.0000000000000082

39. Piovesan A, Berti F, Villa T et al (2019) Computational and experimental fatigue analysis of contoured spinal rods. J Biomech Eng 141(4):044505. https://doi.org/10.1115/1.4042767

40. Berti F, La Barbera L, Piovesan A et al (2018) Residual stresses in titanium spinal rods: effects of two contouring methods and material plastic properties. J Biomech Eng 140(11):111001. https ://doi.org/10.1115/1.4040451

41. La Barbera L, Larson AN, Rawlinson JJ, Aubin CE (2020) In silico patient-specific optimization of correction strategies for thoracic adolescent idiopathic scoliosis. In Press, Clin Biomech. https://doi.org/10.1016/j.clinbiomech.2020.105200

Publisher's Note Springer Nature remains neutral with regard to jurisdictional claims in published maps and institutional affiliations. 